\title{
MAGNETOROTATIONAL CORE-COLLAPSE SUPERNOVAE IN THREE DIMENSIONS
}

\author{
Philipp Mösta ${ }^{1}$, Sherwood Richers ${ }^{1}$, Christian D. OtT $^{1,2,6}$, Roland Haas $^{1}$, Anthony L. Piro ${ }^{1}$, \\ Kristen Boydstun $^{1}$, Ernazar Abdikamalov ${ }^{1}$, Christian Reisswig $^{1,7}$, And Erik SchnetTer ${ }^{3,4,5}$ \\ ${ }^{1}$ TAPIR, Mailcode 350-17, California Institute of Technology, Pasadena, CA 91125, USA; pmoesta @ tapir.caltech.edu \\ ${ }^{2}$ Kavli Institute for the Physics and Mathematics of the Universe (Kavli IPMU WPI), The University of Tokyo, Kashiwa, Japan \\ ${ }^{3}$ Perimeter Institute for Theoretical Physics, Waterloo, ON, Canada \\ ${ }^{4}$ Department of Physics, University of Guelph, Guelph, ON, Canada \\ ${ }^{5}$ Center for Computation \& Technology, Louisiana State University, Baton Rouge, USA \\ Received 2014 March 6; accepted 2014 March 24; published 2014 April 3
}

\begin{abstract}
We present results of new three-dimensional (3D) general-relativistic magnetohydrodynamic simulations of rapidly rotating strongly magnetized core collapse. These simulations are the first of their kind and include a microphysical finite-temperature equation of state and a leakage scheme that captures the overall energetics and lepton number exchange due to postbounce neutrino emission. Our results show that the 3D dynamics of magnetorotational corecollapse supernovae are fundamentally different from what was anticipated on the basis of previous simulations in axisymmetry (2D). A strong bipolar jet that develops in a simulation constrained to $2 \mathrm{D}$ is crippled by a spiral instability and fizzles in full 3D. While multiple (magneto-)hydrodynamic instabilities may be present, our analysis suggests that the jet is disrupted by an $m=1$ kink instability of the ultra-strong toroidal field near the rotation axis. Instead of an axially symmetric jet, a completely new, previously unreported flow structure develops. Highly magnetized spiral plasma funnels expelled from the core push out the shock in polar regions, creating wide secularly expanding lobes. We observe no runaway explosion by the end of the full 3D simulation $185 \mathrm{~ms}$ after bounce. At this time, the lobes have reached maximum radii of $\sim 900 \mathrm{~km}$.
\end{abstract}

Key words: gamma-ray burst: general - instabilities - magnetohydrodynamics (MHD) - neutrinos supernovae: general

Online-only material: color figures

\section{INTRODUCTION}

Stellar collapse liberates gravitational energy of order $10^{53} \mathrm{erg} \mathrm{s}^{-1}$ (100 B). Most (99\%) of that energy is emitted in neutrinos, and the remainder $(\lesssim 1 \mathrm{~B})$ powers a core-collapse supernova (CCSN) explosion. However, a small fraction of CCSNe are hyper-energetic $(\sim 10 \mathrm{~B})$ and involve relativistic outflows (e.g., Soderberg et al. 2006; Drout et al. 2011). These hypernovae come from stripped-envelope progenitors and are classified as Type Ic-bl (H/He deficient, broad spectral lines). Importantly, all $\mathrm{SNe}$ connected with long gamma-ray bursts (GRB) are of Type Ic-bl (Modjaz 2011; Hjorth \& Bloom 2011).

Typical $\mathcal{O}(1) \mathrm{B}$ SNe may be driven by the neutrino mechanism (Bethe \& Wilson 1985), in which neutrinos emitted from the collapsed core deposit energy behind the stalled shock, eventually driving it outward (e.g., Müller et al. 2012; Bruenn et al. 2013). However, the neutrino mechanism appears to lack the efficiency needed to drive hyperenergetic explosions. One possible alternative is the magnetorotational mechanism (e.g., Bisnovatyi-Kogan 1970; LeBlanc \& Wilson 1970; Meier et al. 1976; Wheeler et al. 2002). In its canonical form, rapid rotation of the collapsed core (Period $\mathcal{O}(1) \mathrm{ms}$, spin energy $\mathcal{O}(10) \mathrm{B}$ ), and magnetar-strength magnetic field with a dominant toroidal component drive a strong bipolar jet-like explosion that could result in a hypernova (Burrows et al. 2007).

The magnetorotational mechanism requires rapid precollapse rotation $\left(P_{0} \lesssim 4 \mathrm{~s}\right.$; Ott et al. 2006; Burrows et al. 2007 ) and an efficient process to rapidly amplify the likely weak seed magnetic field of the progenitor. Magnetorotational

\footnotetext{
6 Alfred P. Sloan Research Fellow.

7 NASA Einstein Fellow.
}

instability (MRI, Balbus \& Hawley 1991; Akiyama et al. 2003; Obergaulinger et al. 2009) is one possibility. MRI operates on the free energy of differential rotation and, in combination with dynamo action, has been hypothesized to provide the necessary global field strength on an essentially dynamical timescale (Akiyama et al. 2003; Thompson et al. 2005). The wavelength of the fastest growing MRI mode in a postbounce CCSN core is much smaller than what can currently be resolved in global multi-dimensional CCSN simulations. Under the assumption that MRI and dynamo operate as envisioned, a common approach is to start with a likely unphysically strong precollapse field of $10^{12}-10^{13} \mathrm{G}$. During collapse and early postbounce evolution, this field is amplified by flux compression and rotational winding to dynamically important field strength of $B_{\text {tor }} \gtrsim 10^{15}-10^{16} \mathrm{G}$ (Burrows et al. 2007). In this way, a number of recent two-dimensional (2D) magnetohydrodynamic (MHD) simulations found robust and strong jet-driven explosions (e.g., Shibata et al. 2006; Burrows et al. 2007; Takiwaki \& Kotake 2011), while Sawai \& Yamada (2014) studied a weakly magnetized progenitor. Only a handful of 3D studies were carried out with varying degrees of microphysical realism (Mikami et al. 2008; Kuroda \& Umeda 2010; Scheidegger et al. 2010; Winteler et al. 2012) and none compared 2D and 3D dynamics directly.

In this Letter, we present new full 3D dynamical-spacetime general-relativistic MHD (GRMHD) simulations of rapidly rotating magnetized CCSNe. These are the first to employ a microphysical finite-temperature equation of state, a realistic progenitor model, and an approximate neutrino treatment for collapse and postbounce evolution. We carry out simulations in full unconstrained 3D and compare with simulations starting from identical initial conditions, but constrained to 2D. Our 

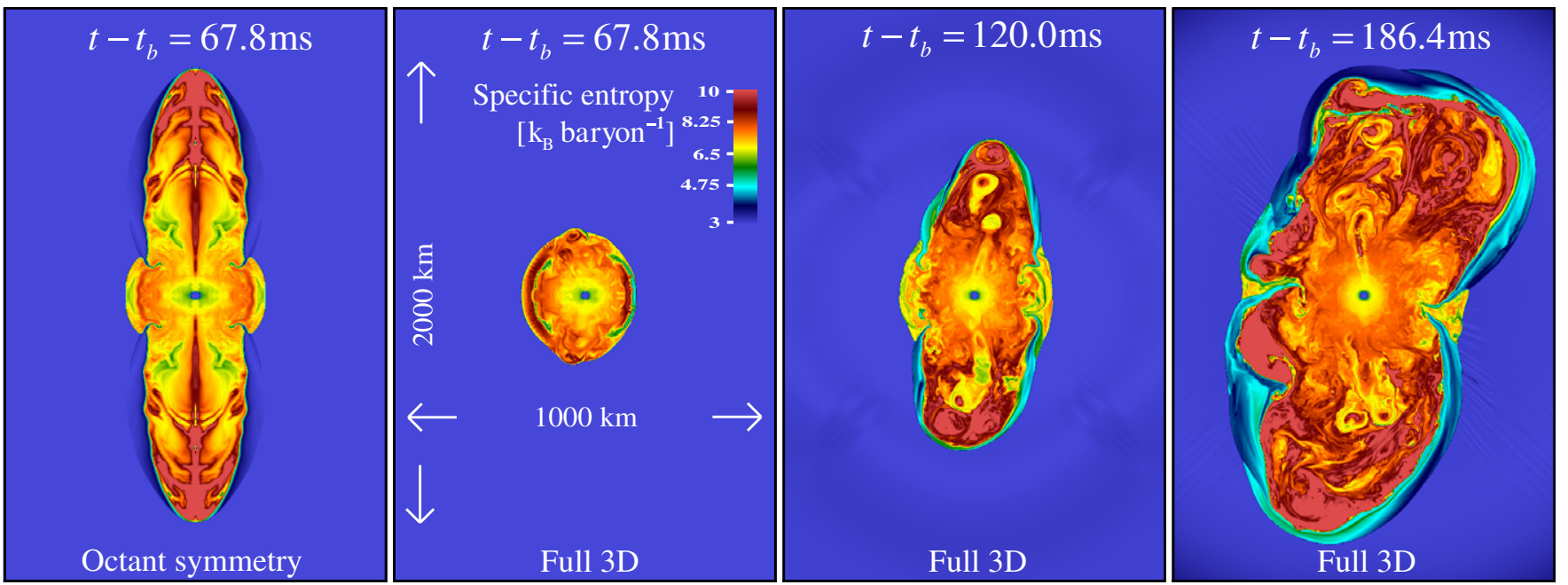

Figure 1. Meridional slices ( $x-z$-plane; $z$ being the vertical) of the specific entropy at various postbounce times. The "2D" (octant 3D) simulation (leftmost panel) shows a clear bipolar jet, while in the full 3D simulation (three panels to the right) the initial jet fails and the subsequent evolution results in large-scale asymmetric lobes.

(A color version of this figure is available in the online journal.)

results for a model with initial poloidal $B$ field of $10^{12} \mathrm{G}$ indicate that 2D and 3D magnetorotational CCSNe are fundamentally different. In 2D, a strong jet-driven explosion is obtained, while in unconstrained 3D, the developing jet is destroyed by nonaxisymmetric dynamics, caused most likely by an $m=1$ MHD kink instability. The subsequent CCSN evolution leads to two large asymmetric shocked lobes at high latitudes. Highly magnetized tubes tangle, twist, and drive the global shock front steadily, but not dynamically outward. Runaway explosion does not occur during the $\sim 185 \mathrm{~ms}$ of postbounce time covered.

\section{METHODS AND SETUP}

We employ ideal GRMHD with adaptive mesh refinement (AMR) and spacetime evolution provided by the open-source EinsteinToolkit (Mösta et al. 2014; Löffler et al. 2012). GRMHD is implemented in a finite-volume fashion with WENO5 reconstruction (Reisswig et al. 2013; Tchekhovskoy et al. 2007) and the HLLE Riemann solver (Einfeldt 1988) and constrained transport (Tóth 2000) for maintaining $\operatorname{div} \boldsymbol{B}=0$. We employ the $K_{0}=220 \mathrm{MeV}$ variant of the equation of state of Lattimer \& Swesty (1991) and the neutrino leakage/heating approximations described in O'Connor \& Ott (2010) and Ott et al. (2012). At the precollapse stage, we cover the inner $\sim 5700 \mathrm{~km}$ of the star with four AMR levels and add five more during collapse. After bounce, the protoneutron star is covered with a resolution of $\sim 370 \mathrm{~m}$ and AMR is set up to always cover the shocked region with at least $1.48 \mathrm{~km}$ linear resolution.

We take the $25 M_{\odot}$ (at zero-age-main-sequence) presupernova model E25 from Heger et al. (2000) and set up axisymmetric precollapse rotation using the rotation law of Takiwaki \& Kotake (2011; see their Equation (1)) with an initial central angular velocity of $2.8 \mathrm{rad} \mathrm{s}^{-1}$. The fall-off in cylindrical radius and vertical position is controlled by the parameters $x_{0}=500 \mathrm{~km}$ and $z_{0}=2000 \mathrm{~km}$, respectively. We set up the initial magnetic field with a vector potential of the form $A_{r}=A_{\theta}=0 ; A_{\phi}=B_{0}\left(r_{0}^{3}\right)\left(r^{3}+r_{0}^{3}\right)^{-1} r \sin \theta$, where $B_{0}$ controls the strength of the field.

In this way, we obtain a modified dipolar field structure that stays nearly uniform in strength within radius $r_{0}$ and falls off like a dipole outside. We set $B_{0}=10^{12} \mathrm{G}$ and choose $r_{0}=1000 \mathrm{~km}$ to match the initial conditions of model B12X5 $\beta 0.1$ of the 2D study of Takiwaki \& Kotake (2011), in which a jet-driven explosion is launched $\sim 20 \mathrm{~ms}$ after bounce.

We perform simulations both in full, unconstrained 3D and in octant symmetry 3D (90 degree rotational symmetry in the $x-y$-plane and reflection symmetry across the $x-y$-plane) with otherwise identical setups. Octant symmetry suppresses most nonaxisymmetric dynamics, since it allows only modes with azimuthal numbers that are multiples of $m=4$. In order to study the impact of potential low-mode nonaxisymmetric dynamics on jet formation, we add a $1 \% m=1$ perturbation (random perturbations lead to qualitatively the same results) to the full 3D run. Focusing on a potential instability of the strong toroidal field near the spin axis, we apply this perturbation to the velocity field within a cylindrical radius of $15 \mathrm{~km}$ and outside the protoneutron star, $30 \mathrm{~km} \leqslant|z| \leqslant 75 \mathrm{~km}, 5 \mathrm{~ms}$ after bounce.

\section{RESULTS}

Collapse and the very early postbounce evolution proceed identically in octant symmetry and full 3D. At bounce, $\sim 350 \mathrm{~ms}$ after the onset of collapse, the poloidal and toroidal $B$ field components reach $B_{\mathrm{pol}}, B_{\mathrm{tor}} \sim 10^{15} \mathrm{G}$. The hydrodynamic shock launched at bounce, still approximately spherical, stalls after $\sim 10 \mathrm{~ms}$ at a radius of $\sim 110 \mathrm{~km}$. Rotational winding, operating on the extreme differential rotation in the region between inner core and shock, amplifies the toroidal component to $10^{16} \mathrm{G}$ near the rotation axis within $\sim 20 \mathrm{~ms}$ of bounce. At this time, the strong polar magnetic pressure gradient, in combination with hoop stresses excerted by the toroidal field, launches a bipolar outflow. As depicted by the leftmost panel of Figure 1, a jet develops and reaches $\sim 800 \mathrm{~km}$ after $\sim 70 \mathrm{~ms}$ in the octant-symmetry run. The expansion speed at that point is mildly relativistic $\left(v_{r} \simeq 0.1-0.15 c\right)$. This is consistent with the 2D findings of Takiwaki \& Kotake (2011).

The full 3D run begins to diverge from its more symmetric counterpart around $\sim 15 \mathrm{~ms}$ after bounce. A nonaxisymmetric spiral $(m=1)$ deformation develops near the rotation axis. It distorts and bends the initially nearly axisymmetrically developing jet, keeping it from breaking out of the stalled shock. The nearly prompt magnetorotational explosion of the 
octant-symmetry run fails in full 3D. The subsequent 3D evolution is fundamentally different from 2D, as evidenced by the three panels of Figure 1 depicting meridional specific entropy slices at different times in the full 3D run. Until $80 \mathrm{~ms}$, the shock remains stalled and nearly spherical. The $m=1$ dynamics pervade the entire postshock region and cause a spiralsloshing of the shock front that is reminiscent of the standingaccretion shock instability in rotating 3D CCSNe (cf. Kuroda et al. 2014). Later, highly magnetized $\left(\beta=P_{\text {gas }} / P_{\text {mag }} \ll 1\right)$ funnels of high-entropy material protrude from polar regions of the core and secularly push out the shock into two dramatic tilted lobes. At the end of our simulation ( $\sim 185 \mathrm{~ms}$ after bounce) the lobes fill polar cones of $\sim 90^{\circ}$ and are only gradually expanding as low- $\beta$ material is pushed out from below. Accreting material is deflected by these lobes and pushed towards the equator where it accretes through the remainder of the initial nearly spherical shock.

\subsection{Nonaxisymmetric Instability and Jet Formation}

The results discussed above suggest that the full 3D run is subject to a spiral instability that grows from $\sim 1 \% \mathrm{~m}=1$ seeds in the velocity field to non-linear scale within the first $\sim 20 \mathrm{~ms}$ after bounce. This instability quenches the jet. Figure 2 depicts the linear growth and non-linear saturation of the spiral instability at various locations along the spin axis outside the protoneutron star.

In the rotating CCSN context, rotational shear instabilities in the protoneutron star (e.g., Ott et al. 2007) and a spiral standing accretion shock instability (SASI; e.g., Kuroda et al. 2014) were discussed to potentially arise already at early postbounce times. It is unlikely that either of these is excited in our simulations, since we choose to perturb a radially and vertically narrow region along the spin axis outside of the protoneutron star and far from the shock, within the region of the highest toroidal magnetic field strength. A spiral MHD instability may thus be the driving agent behind the strong asymmetry in our simulation.

One possible such instability is the screw-pinch kink instability that has been studied in jets from active galactic nuclei (e.g., Begelman 1998; Mignone et al. 2010; McKinney \& Blandford $2009)$. The $B$ field near the spin axis in our simulation can be roughly approximated by a screw-pinch field configuration. This consists of a non-rotating plasma cylinder and a magnetic field of the form

$$
\boldsymbol{B}=B_{\mathrm{tor}}(r) \hat{\phi}+B_{z} \hat{z}
$$

where $\hat{z}$ is along the rotation axis, $\hat{\phi}$ is in the toroidal direction, $B_{z}$ is a constant vertical component of the $B$ field, and $B_{\text {tor }}(r)$ is the radially-varying toroidal component of the $B$ field. We can express perturbations to the jet in the form of fluid displacements as a sum of basis elements of the form $\boldsymbol{\xi}_{k m} \propto e^{i(k z+m \phi-\omega t)}$, where $m$ is an integer, $k$ is the vertical wave number, and $\omega$ is the oscillation frequency of the mode. The Kruskal-Shafranov stability criterion states that a plasma cylinder confined to a finite radius $a$ (as in a tokamak) is unstable to kink ( $m= \pm 1)$ modes if $B_{\text {tor }} / B_{z}>2 \pi a / L$, where $L$ is the length of the cylinder and the sign of $m$ is such that the mode's helicity is opposite to the field helix (Shafranov 1956; Kruskal \& Tuck 1958). Unconfined screw-pinch structures with $B_{\text {tor }} \gg B_{z}$ have been shown to be violently unstable to $m=1$ modes at short vertical wavelengths $(k r \gg 1)$ when $d \ln B_{\text {tor }} / d \ln r>-1 / 2$ and the plasma parameter is sufficiently large $(\beta>2 / 3 \gamma$, where $\gamma$ is the local adiabatic index). Under these conditions (which are
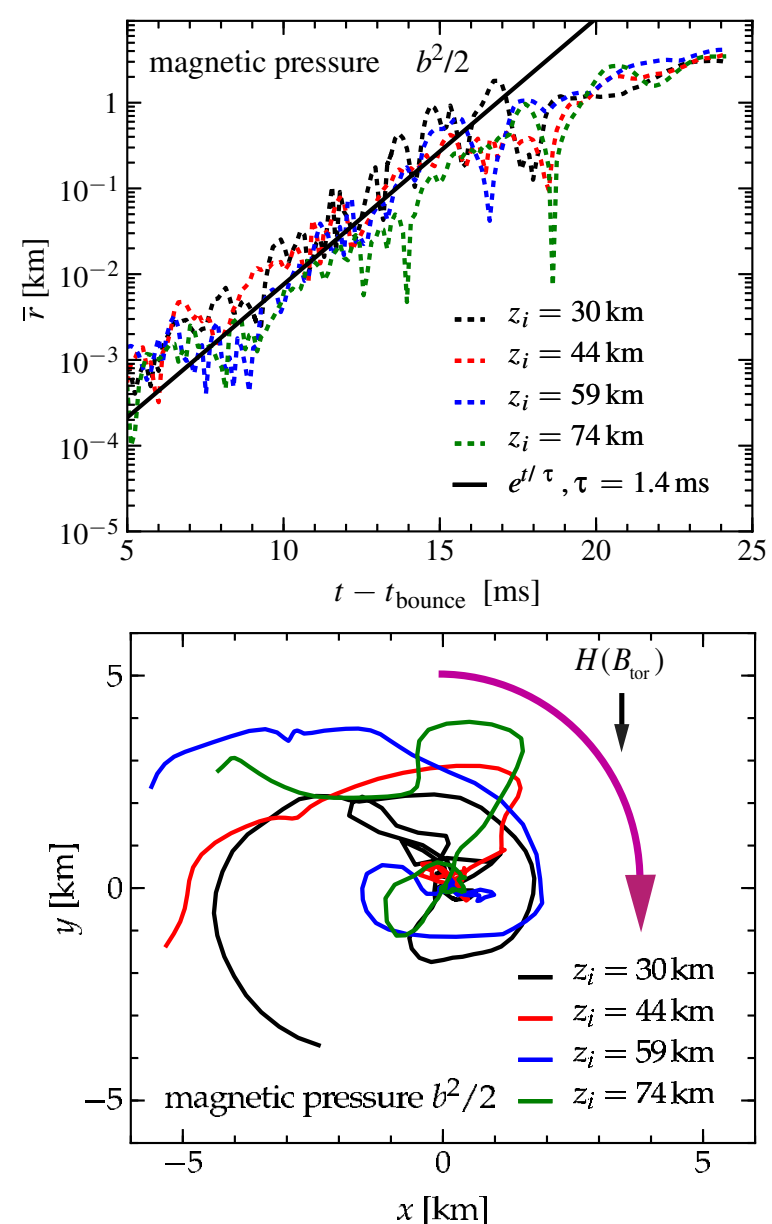

Figure 2. Top panel: barycenter displacement $\bar{r}$ of $b^{2}$ in $x-y$ planes at different heights $z_{i}$. To minimize the influence of material that does not belong to the unstable jet, we include only cells inside a radius of $15 \mathrm{~km}$. We observe exponential growth of the displacement in the early postbounce evolution until saturation at $t-t_{b} \sim 20 \mathrm{~ms}$. The growth rate $\tau_{\text {fgm,sim }} \approx 1.4 \mathrm{~ms}$ is consistent with estimates for the MHD kink instability (see Section 3.1). Bottom panel: tracks of the barycenter in the $x-y$ plane at different $z_{i}$. They are plotted for the interval shown in the top panel. The tracks trace the spiral nature of the displacement. Note that, as required for the perturbation to be unstable (Begelman 1998), the helicity of the displacement motion (counter-clockwise in the $x-y$ plane) is opposite to the helicity of the toroidal magnetic field $H\left(B_{\text {tor }}\right)$ (clockwise in the $x-y$-plane, magenta arrow).

(A color version of this figure is available in the online journal.)

only approximately met in our simulation), the fastest growing unstable mode (fgm) is amplified on a timescale comparable to the Alfvén travel time around a toroidal loop (Begelman 1998). The expected $m=1$ growth timescale and vertical wavelength in the most unstable regions of the jet $\sim 10-15 \mathrm{~ms}$ after bounce are

$$
\tau_{\mathrm{fgm}} \approx \frac{4 a \sqrt{\pi \rho}}{B_{\mathrm{tor}}} \approx 1 \mathrm{~ms}, \quad \lambda_{\mathrm{fgm}} \approx \frac{4 \pi a B_{z}}{B_{\mathrm{tor}}} \approx 5 \mathrm{~km},
$$

where $a$ is the radius of the most unstable region. The growth time is much shorter than the time it would take for the jet to propagate through the shocked region.

The effect of the kink instability can be most clearly seen in a displacement of the jet barycenter away from the rotation axis of the core. We measure the displacement of the jet in our full 3D run by computing the barycenter displacement (planar "center-of-mass" displacement; Mignone et al. 2010) of the 

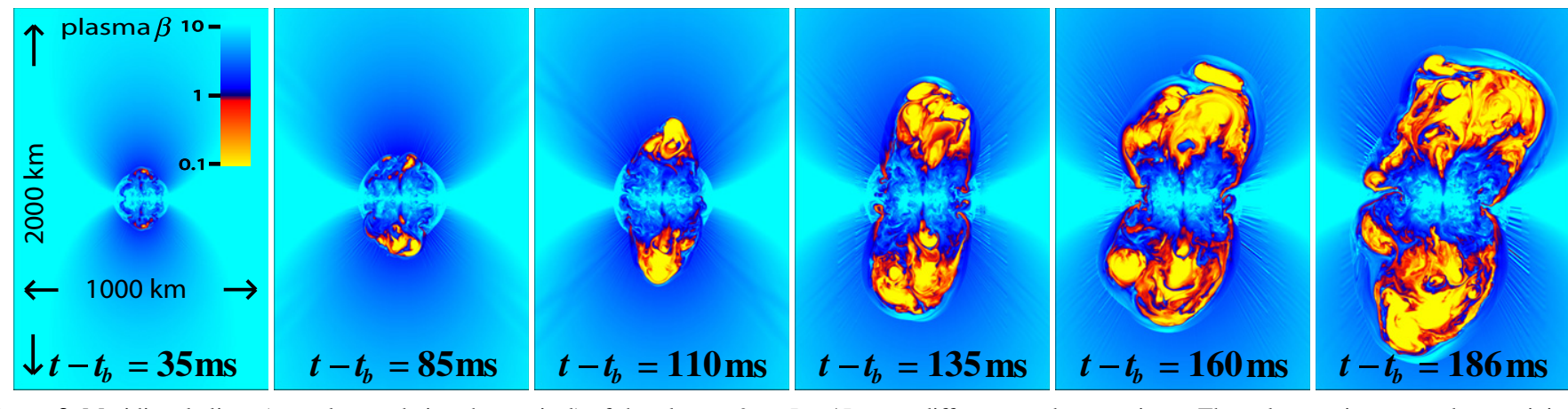

Figure 3. Meridional slices ( $x-z$-plane; $z$ being the vertical) of the plasma $\beta=P_{\text {gas }} / P_{\text {mag }}$ at different postbounce times. The colormap is saturated at a minimum $\beta=0.01$ and a maximum $\beta=10$. Regions of $\beta<1$ (warm colors, magnetically dominated) are underdense due to expansion from magnetic pressure, rise buoyantly, and push out the shock front in two prominent polar lobes.

(A color version of this figure is available in the online journal.)

co-moving magnetic field strength $b^{2}$ (see, e.g., Mösta et al. 2014) in $x y$-slices at different heights $z_{i}$ along the rotation axis (Figure 2). $b^{2}$ probes the MHD effects in our simulations most directly, but other variable choices, e.g., the specific entropy $s$, exhibit similar behavior as flux freezing couples fluid properties to the magnetic field evolution. Figure 2 demonstrates that the jet experiences significant displacements from the rotation axis of the core in a spiraling motion with helicity opposite to that of the magnetic field (indicated by the magenta colored arrow in Figure 2), and that the growth rate and dominant instability length scale roughly agree with those predicted by a kink unstable jet in our analysis.

\subsection{Magnetized Expanding Lobes}

Although the initial bipolar jet fails to promptly break out of the stalled shock, MHD becomes dominant tens of milliseconds later. Starting around $\sim 80 \mathrm{~ms}$ after bounce, outflows of highly magnetized material are continuously launched from the protoneutron star and propagate along the rotation axis of the core. This is depicted in Figure 3, which presents meridional slices of the plasma parameter $\beta$ at a range of postbounce times. The highly magnetized (low- $\beta$ ) material does not stay neatly confined to the rotation axis.

In Figure 4, we present volume renderings of the specific entropy and plasma parameter $\beta 161 \mathrm{~ms}$ after bounce. These volume renderings show the full truth of how outflows driven by the core are severely deformed, sheared, and wound up as they propagate in the $z$-direction. The material that is expelled from the vicinity of the protoneutron star forms tube-like structures that are highly magnetized $(\beta \sim 0.01-0.1)$, underdense $(\sim 1 \%$ the density of the surrounding fluid), and rise buoyantly. The overall structure of the shocked region evolves toward strongly prolate-shape with two, roughly $90^{\circ}$ filling tilted lobes at both poles (cf. Figures 1, 3, and 4).

The lobes secularly expand to $\sim 900 \mathrm{~km}$ during the simulated time, but their expansion never becomes dynamical. Accreting material is funneled to equatorial regions where it continues to settle onto the protoneutron star. The $B$-field geometry in the later evolution corresponds to that of a tightly wound coil close to the protoneutron star, but the field lines open up in a spiraling fashion further out, yet still behind the shock. This is consistent with magnetized material moving away from the rotation axis as it propagates in the general $z$-direction. In 2D simulations, a confining magnetic-tower structure forms instead (Burrows et al. 2007).

\section{DISCUSSION}

Our results show that 3D magnetorotational core-collapse supernovae are fundamentally different from what has been anticipated on the basis of axisymmetric simulations (Burrows et al. 2007; Dessart et al. 2008; Takiwaki \& Kotake 2011). A jet that develops in $2 \mathrm{D}$ is disrupted and fizzles in $3 \mathrm{D}$. We suggest that the instability driving this is most likely an MHD kink ( $m=1)$ instability to which the toroidally dominated postbounce magnetic-field configuration is prone. Instead of an axially symmetric jet, a completely new, wide double-lobed flow pattern develops, but we obtain no runaway explosion during the simulated time.

The high precollapse field strength of $10^{12} \mathrm{G}$ yields $\sim 10^{16} \mathrm{G}$ in toroidal field and $\beta=P_{\text {gas }} / P_{\text {mag }}<1$ within only $\sim 10-15 \mathrm{~ms}$ of bounce, creating conditions favorable for jet formation. Yet, the growth time of the kink instability is shorter than the time it takes for the jet to develop. In a short test simulation with an even more unrealistic, ten times stronger initial field, a successful jet is launched promptly after bounce (consistent with Winteler et al. 2012, who used a similarly strong field), but subsequently also experiences a spiral displacement.

Realistic precollapse iron cores are not expected to have magnetic fields in excess of $\sim 10^{8}-10^{9} \mathrm{G}$, which may be amplified to no more than $\sim 10^{12} \mathrm{G}$ during collapse (Burrows et al. 2007). The $10^{15}-10^{16} \mathrm{G}$ of large-scale toroidal field required to drive a magnetorotational jet must be built up after bounce. This will likely require tens to hundreds of dynamical times, even if the magnetorotational instability operates in conjunction with a dynamo. The results of the present and previous full 3D rotating CCSN simulations (Ott et al. 2007; Kuroda et al. 2014) suggest that MHD and also a variety of nonaxisymmetric hydrodynamic instabilities will grow to nonlinear regimes on shorter timescales, disrupting any possibly developing axial outflow. This is why we believe that the dynamics and flow structures seen in our full $3 D$ simulation may be generic to the postbounce evolution of rapidly rotating magnetized core collapse that starts from realistic initial conditions.

If the polar lobes eventually accelerate, the resulting explosion will be asymmetric, though probably less so than jet-driven explosion. The lobes carry neutron-rich $\left(Y_{e} \sim 0.1-0.2\right)$ material of moderate entropy $\left(s \sim 10-15 k_{B}\right.$ baryon $\left.^{-1}\right)$, which could lead to interesting $r$-process yields, similar to what Winteler et al. (2012) found for their prompt jet-driven explosion. Even if the lobes continue to move outward, accretion in equatorial regions may continue, eventually (after $2-3 \mathrm{~s}$ ) leading to the 

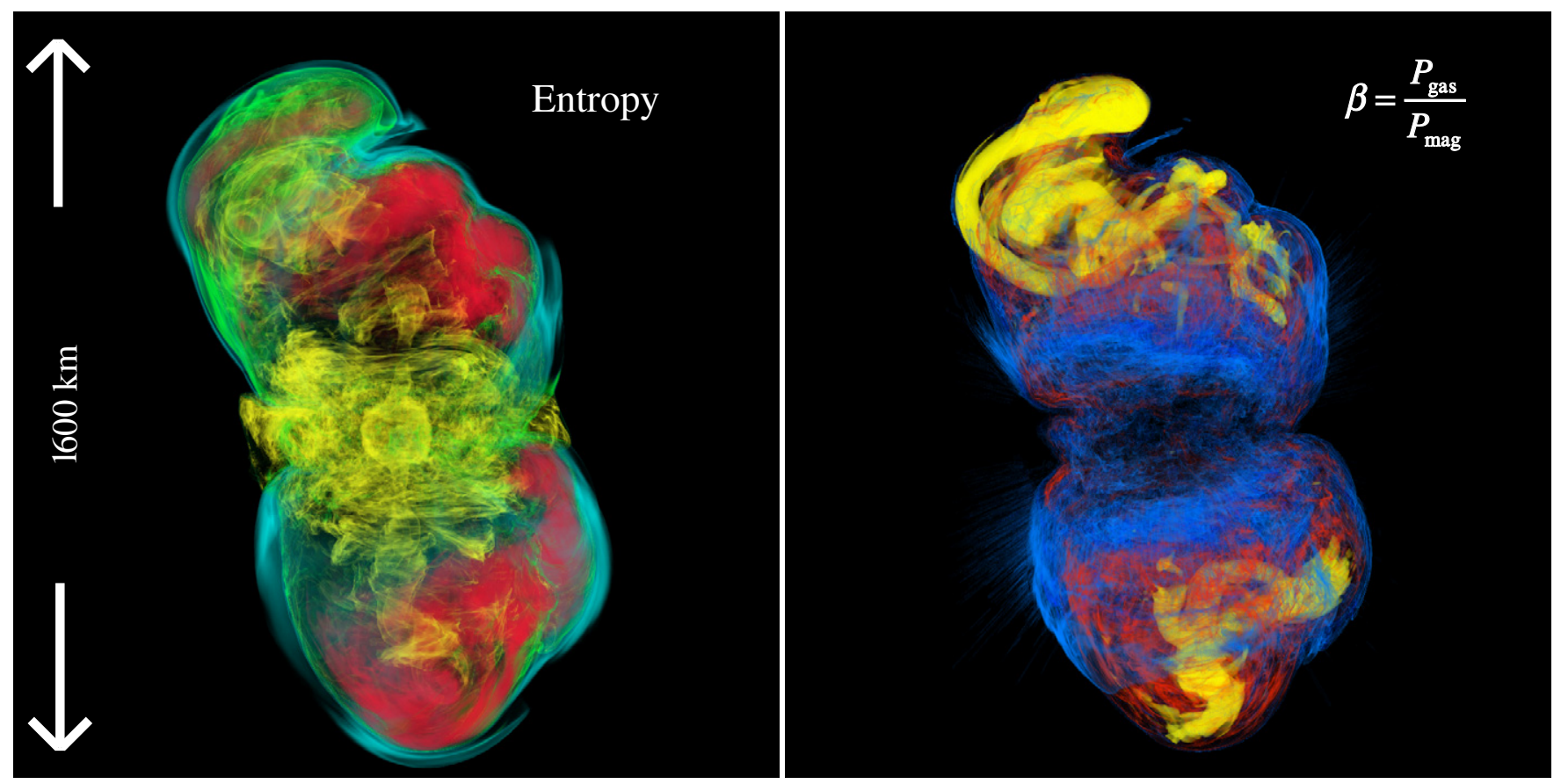

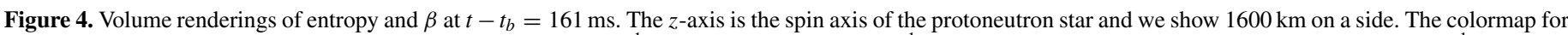

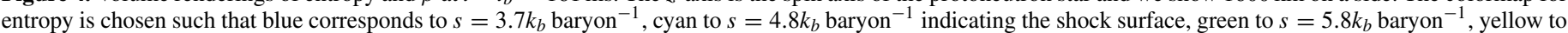

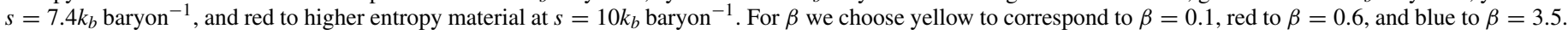

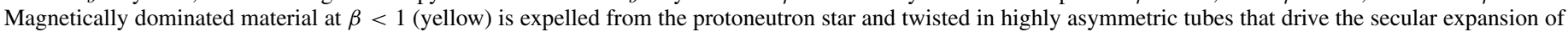
the polar lobes.

(A color version of this figure is available in the online journal.)

collapse of the protoneutron star and black hole formation. In this case, the engine supplying the lobes with low- $\beta$ plasma is shut off. Unless their material has reached positive total energy, the lobes will fall back onto the black hole, which will subsequently hyperaccrete until material becomes centrifugally supported in an accretion disk. This would set the stage for a subsequent long GRB and an associated Type Ic-bl CCSN that would be driven by a collapsar central engine (Woosley 1993) rather than by a protomagnetar (Metzger et al. 2011).

The results of the present study highlight the importance of studying magnetorotational CCSNe in 3D. Future work will be necessary to explore later postbounce dynamics, the sensitivity to initial conditions and numerical resolution, and possible nucleosynthetic yields. Animations and further details on our simulations are available at http://stellarcollapse.org/cc3dgrmhd.

The authors would like to thank A. Burrows, S. Couch, U. Gamma, D. Meier, and L. Roberts for discussions. This research was partially supported by NSF grants AST-1212170, PHY1151197, and OCI-0905046, and the Sherman Fairchild Foundation. S.R. is supported by a DOE Computational Science Graduate Fellowship DE-FG02-97ER25308. C.R. acknowledges support by NASA through Einstein Fellowship grant PF2-130099. The simulations were carried out on XSEDE (TG-PHY100033) and on NSF/NCSA BlueWaters (PRAC OCI-0941653).

\section{REFERENCES}

Akiyama, S., Wheeler, J. C., Meier, D. L., \& Lichtenstadt, I. 2003, ApJ, 584,954

Balbus, S. A., \& Hawley, J. F. 1991, ApJ, 376, 214

Begelman, M. C. 1998, ApJ, 493, 291

Bethe, H. A., \& Wilson, J. R. 1985, ApJ, 295, 14

Bisnovatyi-Kogan, G. S. 1970, AZh, 47, 813
Bruenn, S. W., Mezzacappa, A., Hix, W. R., et al. 2013, ApJL, 767, L6 Burrows, A., Dessart, L., Livne, E., Ott, C. D., \& Murphy, J. 2007, ApJ, 664,416

Dessart, L., Burrows, A., Livne, E., \& Ott, C. D. 2008, ApJL, 673, L43

Drout, M. R., Soderberg, A. M., Gal-Yam, A., et al. 2011, ApJ, 741, 97

Einfeldt, B. 1988, in Shock Tubes and Waves, Proc. 16th Int. Symp., Aachen, Germany, July 26-31, 1987 (Weinheim: VCH Verlag), 671

Heger, A., Langer, N., \& Woosley, S. E. 2000, ApJ, 528, 368

Hjorth, J., \& Bloom, J. S. 2011, in Gamma-Ray Bursts, ed. C. Kouveliotou, R. A. M. J. Wijers, \& S. E. Woosley (Cambridge: Cambridge Univ. Press)

Kruskal, M., \& Tuck, J. L. 1958, RSPSA, 245, 222

Kuroda, T., Takiwaki, T., \& Kotake, K. 2014, PhRvD, 89, 044011

Kuroda, T., \& Umeda, H. 2010, ApJS, 191, 439

Lattimer, J. M., \& Swesty, F. D. 1991, NuPhA, 535, 331

LeBlanc, J. M., \& Wilson, J. R. 1970, ApJ, 161, 541

Löffler, F., Faber, J., Bentivegna, E., et al. 2012, CQGra, 29, 115001

McKinney, J. C., \& Blandford, R. D. 2009, MNRAS, 394, L126

Meier, D. L., Epstein, R. I., Arnett, W. D., \& Schramm, D. N. 1976, ApJ, 204, 869

Metzger, B. D., Giannios, D., Thompson, T. A., Bucciantini, N., \& Quataert, E. 2011, MNRAS, 413, 2031

Mignone, A., Rossi, P., Bodo, G., Ferrari, A., \& Massaglia, S. 2010, MNRAS, 402, 7

Mikami, H., Sato, Y., Matsumoto, T., \& Hanawa, T. 2008, ApJ, 683, 357

Modjaz, M. 2011, AN, 332, 434

Mösta, P., Mundim, B. C., Faber, J. A., et al. 2014, CQGra, 31, 015005

Müller, B., Janka, H.-T., \& Marek, A. 2012, ApJ, 756, 84

Obergaulinger, M., Cerdá-Durán, P., Müller, E., \& Aloy, M. A. 2009, A\&A, 498, 241

O'Connor, E., \& Ott, C. D. 2010, CQGra, 27, 114103

Ott, C. D., Abdikamalov, E., O’Connor, E., et al. 2012, PhRvD, 86, 024026

Ott, C. D., Burrows, A., Thompson, T. A., Livne, E., \& Walder, R. 2006, ApJS, 164,130

Ott, C. D., Dimmelmeier, H., Marek, A., et al. 2007, PhRvL, 98, 261101

Reisswig, C., Haas, R., Ott, C. D., et al. 2013, PhRvD, 87, 064023

Sawai, H., \& Yamada, S. 2014, ApJL, 784, L10

Scheidegger, S., Käppeli, R., Whitehouse, S. C., Fischer, T., \& Liebendörfer, M. 2010, A\&A, 514, A51

Shafranov, V. 1956, At. Energy, 5, 38 
Shibata, M., Liu, Y. T., Shapiro, S. L., \& Stephens, B. C. 2006, PhRvD, 74 104026

Soderberg, A. M., Kulkarni, S. R., Nakar, E., et al. 2006, Natur, 442, 1014

Takiwaki, T., \& Kotake, K. 2011, ApJ, 743, 30

Tchekhovskoy, A., McKinney, J. C., \& Narayan, R. 2007, MNRAS, 379, 469
Thompson, T. A., Quataert, E., \& Burrows, A. 2005, ApJ, 620, 861 Tóth, G. 2000, JCoPh, 161, 605

Wheeler, J. C., Meier, D. L., \& Wilson, J. R. 2002, ApJ, 568, 807

Winteler, C., Käppeli, R., Perego, A., et al. 2012, ApJL, 750, L22 Woosley, S. E. 1993, ApJ, 405, 273 